\title{
Kuga-Satake construction and cohomology of hyperkähler manifolds
}

\author{
Nikon Kurnosov ${ }^{1}$, Andrey Soldatenkov², Misha Verbitsky ${ }^{3}$
}

\begin{abstract}
Let $M$ be a simple hyperkähler manifold. Kuga-Satake construction gives an embedding of $H^{2}(M, \mathbb{C})$ into the second cohomology of a torus, compatible with the Hodge structure. We construct a torus $T$ and an embedding of the graded cohomology space $H^{\bullet}(M, \mathbb{C}) \rightarrow H^{\bullet+l}(T, \mathbb{C})$ for some $l$, which is compatible with the Hodge structures and the Poincaré pairing. Moreover, this embedding is compatible with an action of the Lie algebra generated by all Lefschetz $\mathfrak{s l}(2)$-triples on $M$.
\end{abstract}

\section{Contents}

1 Introduction $\quad \mathbf{2}$

1.1 Kuga-Satake construction and $k$-symplectic geometry . . . . . . . 2

1.2 Hyperkähler manifolds . . . . . . . . . . . . . . . . . 4

2 Graded Frobenius algebras and $\mathfrak{s l}(2)$-triples $\quad 5$

2.1 Frobenius algebras . . . . . . . . . . . . . 5

$2.2 \quad F$-algebras . . . . . . . . . . . . . . . 7

3 Clifford modules and the Kuga-Satake construction $\quad 8$

3.1 Clifford algebras . . . . . . . . . . . . . . 8

3.2 Invariant forms on $\mathcal{C}$-modules . . . . . . . . . . . . . . . . 9

3.3 The embedding . . . . . . . . . . . . . . . . . . . . . . . 10

3.4 Mukai extension . . . . . . . . . . . . . . . . . . 13

3.5 The Lie algebra action . . . . . . . . . . . . . . . 13

4 Multidimensional Kuga-Satake construction $\quad 17$

4.1 The embedding . . . . . . . . . . . . . . . . . 17

4.2 Polarization . . . . . . . . . . . . . . . . . . . 18

\footnotetext{
${ }^{1}$ Partially supported by the Russian Academic Excellence Project '5-100' within AG Laboratory NRU-HSE and by the contest "Young Russian Mathematics".

${ }^{2}$ Partially supported by the SFB/TR 45 "Periods, Moduli Spaces and Arithmetic of Algebraic Varieties" of the DFG (German Research Foundation).

${ }^{3}$ Partially supported by the Russian Academic Excellence Project '5-100' within AG Laboratory NRU-HSE, FAPERJ E-26/202.912/2018 and CNPq - Process 313608/2017-2
} 
N. Kurnosov, A. Soldatenkov, M. Verbitsky Kuga-Satake construction and cohomology of HK manifolds

A Supersymmetry in hyperkähler geometry $\quad 19$

A.1 Supersymmetry on Kähler manifolds . . . . . . . . . . . . . . . . 19

A.2 Supersymmetry on hyperkähler manifolds . . . . . . . . . . . 20

A.3 Lie algebra generated by all $\mathfrak{s o}(4,1) \ldots \ldots . \ldots . . \ldots 21$

\section{Introduction}

\subsection{Kuga-Satake construction and $k$-symplectic geom- etry}

The classical Kuga-Satake construction [KS], introduced by Ichiro Satake and Michio Kuga in 1967, is used to construct a holomorphic embedding of the space of Hodge structures of K3 type (identified with the bounded Hermitian symmetric domain of Cartan type IV) into the space of Hodge structures of abelian varieties, identified with the bounded Hermitian symmetric domain of Cartan type III. The construction attaches to a Hodge structure $H$ of K3 type a Hodge structure $V$ of weight one, where $V$ is the even part of the Clifford algebra of $H$ with the intersection form. This way, the Kuga-Satake construction produces an abelian variety from a given polarized K3 surface. More generally, from a non-algebraic K3 surface the construction produces a compact complex torus.

We give an interpretation of the Kuga-Satake construction based on the theory of $k$-symplectic spaces. Our main result is Theorem 4.1.

The $k$-symplectic spaces were introduced in [SV2]; however the corresponding geometric structure for $k=3$ was known since 1990-ies under the name "hypersymplectic". A hypersymplectic space ([DS]) is a vector space $V$ over a field $k=\mathbb{R}$ or $\mathbb{C}$ equipped with a triple of symplectic forms $\omega_{1}, \omega_{2}, \omega_{3}$ in such a way that the operators $\omega_{i} \circ \omega_{j}^{-1}: V \longrightarrow V$ generate the matrix algebra $\operatorname{Mat}(2, \mathrm{k})$.

Hypersymplectic manifolds are manifolds equipped with a triple of symplectic forms giving it a hypersymplectic structure at each tangent space. They were introduced by N. Hitchin [Hi], because the hyperkähler reduction can be naturally extended to the hypersymplectic case. In fact, the hypersymplectic manifolds admit a unique torsion-free connection preserving the triple of symplectic forms (see [JV1]). As shown in [JV1], any hyperkähler manifold $M$ admits a natural complexification $M_{\mathbb{C}}$, which is realized as a component in the moduli of rational curves in its twistor space, and this space is a complex hypersymplectic manifold.

In the paper $[\mathrm{Ka}]$, H. Kamada classified hypersymplectic structures on 
compact surfaces, and gave interesting examples of hypersymplectic structures on a Kodaira surface. ${ }^{1}$

In [JV2], Hitchin's construction for hyperkähler and hypersymplectic reduction was applied to a complex hypersymplectic manifold obtained as a component in the space of rational curves in the twistor space of a hyperkähler manifold. This procedure was called trihyperkähler reduction.

In [JV2], trihyperkähler reduction was applied to the space of geometric instantons on $\mathbb{C} P^{3}$. Using this method it was shown that the moduli of geometric instantons in $\mathbb{C} P^{3}$ is smooth; this solves a longstanding conjecture by W. Barth.

In hyperkähler and real hypersymplectic case, the moment map associated with the $G$-action takes values in $\mathfrak{g}^{*} \otimes_{\mathbb{R}} \mathbb{R}^{3}$, where $\mathfrak{g}^{*}$ is the dual space to the Lie algebra of $G$. For the trihyperkähler reduction, the moment map takes values in $\mathfrak{g}^{*} \otimes_{\mathbb{R}} \mathbb{R}^{7}$, suggesting that there are higher analogues of symplectic, trisymplectic and trihyperkähler structures for which the geometric reduction decreases the dimension by $2^{k} \operatorname{dim} \mathfrak{g}$; this higher analogues should be associated with stable bundles on $\mathbb{C} P^{n}, n>3$, in the same way as the hypersymplectic structures are associated to the instantons on $\mathbb{C} P^{3}$.

Hypersymplectic structures were generalized to $k$-symplectic structures in [SV2]. The starting point was again hyperkähler geometry, but an entirely different facet. Consider a compact complex torus $T$ in a sufficiently general deformation of a complex manifold $M$ of hyperkähler type. In [SV2] it was shown that the first homology $H_{1}(T, \mathbb{R})$ is equipped with a $k$-symplectic structure, where $k=b_{2}(M)$. The intuition behind this construction is very simple. Consider a general triple of cohomology classes $w_{1}, w_{2}, w_{3} \in H^{2}(M)$; it is not hard to see that their restrictions to $H^{2}(T)$, considered as 2 -forms on $H_{1}(T)$, form a hypersymplectic structure.

This is the intuition which underlies the notion of a $k$-symplectic structure, and here is its formal definition.

Definition 1.1: Let $V$ be a vector space, and $\Omega \subset \Lambda^{2} V^{*}$ a subspace of dimension $k$. We say that $\Omega$ is a $k$-symplectic structure if the following two properties are satisfied

(i) For any non-zero $w \in \Omega$, the form $w$ has maximal rank, or its rank is $\frac{1}{2} \operatorname{dim} V$. This implies, in particular, that the set of degenerate forms $\omega \in \Omega$ is a quadric (see [SV2]; this is an easy exercise in linear algebra).

\footnotetext{
${ }^{1}$ Kodaira surface is a non-Kähler compact complex surface obtained as an isotrivial holomorphic elliptic fibration over an elliptic curve.
} 
N. Kurnosov, A. Soldatenkov, M. Verbitsky Kuga-Satake construction and cohomology of HK manifolds

(ii) The quadric $Q \subset \mathbb{P}(\Omega)$ consisting of all degenerate forms $\omega \in \Omega$ is non-degenerate.

It is still unknown what kind of geometric structure is given by a $k$ symplectic analogue of a hypersymplectic manifold, when we are given $k$ symplectic forms on a manifold $M$ satisfying the $k$-symplectic properties at each tangent space $T_{x} M$.

\subsection{Hyperkähler manifolds}

For the convenience of the reader we will recall below the main definitions related to hyperkähler manifolds. For more details see [GHJ] and [Bes].

Definition 1.2: (E. Calabi, $[\mathrm{C}])$ Let $(M, g)$ be a Riemannian manifold with three integrable complex structure operators $I, J, K \in \operatorname{End}(T M)$, satisfying the quaternionic relations $I^{2}=J^{2}=K^{2}=I J K=-\mathrm{Id}$. Suppose that $g$ is Kähler with respect to $I, J$ and $K$. Then $(M, I, J, K, g)$ is called hyperkähler.

Definition 1.3: A holomorphically symplectic manifold is a complex manifold equipped with a non-degenerate, holomorphic $(2,0)$-form.

The metric $g$ on a hyperkähler manifold $M$ is Kähler with respect to the complex structures $I, J$ and $K$. Hence we have the Kähler forms $\omega_{I}, \omega_{J}$, $\omega_{K}$, each of them of type $(1,1)$ with respect to the corresponding complex structure. One can check that the form $\Omega_{I}:=\omega_{J}+\sqrt{-1} \omega_{K}$ is a holomorphic symplectic $(2,0)$-form on $(M, I)$. This shows that every hyperkähler manifold is holomorphically symplectic. The partial converse to this statement follows from Yau's solution of the Calabi conjecture (see $[\mathrm{Y}]$ ):

Theorem 1.4: Let $M$ be a compact holomorphically symplectic Kähler manifold. Then $M$ admits a hyperkähler metric which is uniquely determined by the cohomology class of its Kähler form.

In this paper we will consider only compact Kähler holomorphically symplectic manifolds. There is the following structure theorem for such manifolds.

Definition 1.5: A compact hyperkähler manifold $M$ is of maximal holonomy, or simple, or IHS, if $\pi_{1}(M)=0, H^{2,0}(M)=\mathbb{C}$. 
N. Kurnosov, A. Soldatenkov, M. Verbitsky Kuga-Satake construction and cohomology of HK manifolds

Theorem 1.6: (Bogomolov's decomposition: [Bo1]) Any compact hyperkähler manifold admits a finite étale covering which is a product of a torus and several maximal holonomy hyperkähler manifolds. The maximal holonomy hyperkähler components of this decomposition are uniquely determined.

Further on, all hyperkähler manifolds are tacitly assumed to be of maximal holonomy. The following theorem is crucial for the study of cohomology algebras of IHS manifolds.

Theorem 1.7: (Fujiki, $[\mathrm{F}]$ ) Let $M$ be an IHS manifold, $\operatorname{dim}_{\mathbb{C}} M=2 n$. Then there exist a non-degenerate primitive integer quadratic form $q$ on $H^{2}(M, \mathbb{Z})$ and a rational constant $c$, such that for any $\eta \in H^{2}(M, \mathbb{C})$ we have $\int_{M} \eta^{2 n}=c q(\eta, \eta)^{n}$.

Definition 1.8: The quadratic form $q$ from the theorem above is called the Bogomolov-Beauville-Fujiki (BBF) form.

The BBF form is determined up to a sign by the relation from Fujiki's

theorem. The sign may be determined by the condition $q(\Omega, \bar{\Omega})>0$, where $\Omega$ is the holomorphic symplectic form. It is known that the form $q$ has signature $\left(3, b_{2}(M)-3\right)$. It is negative definite on $\omega$-primitive forms, and positive definite on $\langle\Omega, \bar{\Omega}, \omega\rangle$, where $\omega$ is a Kähler form.

\section{$2 \quad$ Graded Frobenius algebras and $\mathfrak{s l}(2)$-triples}

To relate $k$-symplectic structures and the Kuga-Satake construction, we use the Lie algebra action generated by all Lefschetz triples on the cohomology of a hyperkähler manifold. This action was discovered and studied in [LL, $\mathrm{V} 1, \mathrm{~V} 2]$. Let us now recall the main elements of these works.

\subsection{Frobenius algebras}

Definition 2.1: Let $A^{\bullet}=\bigoplus_{i=0}^{r} A^{i}$ be a graded-commutative algebra with $\operatorname{dim} A^{r}=1$. Consider the $A^{r}$-valued form on $A^{\bullet}$ mapping $x, y \in A^{\bullet}$ to the $A^{r}$-component of $x y$. The algebra $A^{\bullet}$ is called degree $r$ graded Frobenius algebra if this pairing is non-degenerate. 
N. Kurnosov, A. Soldatenkov, M. Verbitsky Kuga-Satake construction and cohomology of HK manifolds

The basic example of a Frobenius algebra is the cohomology algebra of a compact manifold.

A Lefschetz triple in a Frobenius algebra $A=\bigoplus_{i=0}^{2 n} A^{i}$ is a triple of operators $\mathrm{L}_{\eta}, \Xi, \Lambda_{\eta} \in \operatorname{End}\left(A^{\bullet}\right)$ where $\eta \in A^{2}$ is a fixed element, $\mathrm{L}_{\eta}(x):=$ $\eta x,\left.\Xi\right|_{A^{i}}=i-n$ and $\Lambda_{\eta}$ is an element such that $\mathrm{L}_{\eta}, \Xi, \Lambda_{\eta}$ form an $\mathfrak{s l}(2)$ triple. It is easy to see that such $\Lambda_{\eta}$ is uniquely determined by $\Xi$ and $\eta$ (this statement is sometimes called "Morozov's lemma", and sometimes included in the statement of Jacobson-Morozov theorem). Existence of one Lefschetz triple is a non-trivial condition; however, the space of $\eta \in A^{2}$ for which the Lefschetz triple exists is Zariski open.

Definition 2.2: The Frobenius-Lefschetz algebra is a Frobenius algebra admitting a Lefschetz $\mathfrak{s l}(2)$-triple.

Remark 2.3: Let $A^{\bullet}$ be a Frobenius-Lefschetz algebra. Consider the Lie algebra $\mathfrak{g}\left(A^{\bullet}\right) \subset \operatorname{End}\left(A^{\bullet}\right)$ generated by all $\mathfrak{s l}(2)$-triples. It was computed for the cohomology of hyperkähler manifolds in [V1, LL] and for other geometric examples of Frobenius algebras (flag varieties, Hodge classes on an abelian variety) in [LL].

Theorem 2.4: Let $M$ be a hyperkähler manifold of maximal holonomy, $A^{\bullet}$ its cohomology algebra and $\mathfrak{g}_{\text {tot }}(M):=\mathfrak{g}\left(A^{\bullet}\right)$ the Lie algebra generated by all Lefschetz $\mathfrak{s l}(2)$-triples. Then $\mathfrak{g}_{\text {tot }}(M)$ is isomorphic to $\mathfrak{s o}\left(4, b_{2}(M)-2\right)$.

Proof: See [LL] or the proof of Theorem A.10; an explicit description of this algebra is also given there.

An important property of the Lie algebra $\mathfrak{g}_{\text {tot }}(M) \cong \mathfrak{s o}\left(4, b_{2}(M)-2\right)$ acting on cohomology of a hyperkähler manifold is that it contains the Weil operators $W_{I}$ for all complex structures of hyperkähler type on $M$ (see Subsection A.2). Recall that for a compact Kähler manifold $(M, I)$ the Weil operator $W_{I} \in \operatorname{End}\left(H^{\bullet}(M)\right)$ acts on $H^{p, q}(M)$ as $\sqrt{-1}(p-q)$.

This implies that the Hodge structure on $H^{\bullet}(M, \mathbb{C})$ is induced by the action of $\mathfrak{g}_{\text {tot }}(M)$. We will use this observation to construct the Kuga-Sataketype embedding of the cohomology of $M$ into the cohomology of a complex torus (see Section 3 and Theorem 4.1). The construction essentially consists of three steps:

1. Start from a hyperkähler manifold of maximal holonomy, and let $H=$ $H^{2}(M, \mathbb{C})$ be its second cohomology space equipped with the BBF form. 
N. Kurnosov, A. Soldatenkov, M. Verbitsky Kuga-Satake construction and cohomology of HK manifolds

Construct a $k$-symplectic space $V$ such that $\varphi: H \hookrightarrow \Lambda^{2} V^{*}$ is its $k$ symplectic structure. Both $V$ and $\varphi$ will be naturally defined over $\mathbb{Q}$. We will interpret $\Lambda^{2} V^{*}$ as the second cohomology of a complex torus $T$ well-defined up to isogeny.

2. Let $\mathfrak{g}(H) \subset \mathfrak{g}_{\text {tot }}(T)$ be the subalgebra generated by the image of $H$ in $\Lambda^{2} V^{*}$. Then $\mathfrak{g}(H) \simeq \mathfrak{g}_{\text {tot }}(M)$. Consider the action of $\mathfrak{g}(H)$ on $H^{\bullet}(T, \mathbb{C})$. Let $\mathfrak{g}_{0}(H) \subset \mathfrak{g}(H)$ be the set of all elements which preserve the grading. By construction, the embedding $\varphi$ is $\mathfrak{g}_{0}(H)$-invariant. Since the Weil endomorphisms of $H^{2}(T, \mathbb{C})$ and of $H$ belong to $\mathfrak{g}(H)$, the embedding $\varphi$ is compatible with the Hodge structure.

3. One can choose $V$ in such a way that the $\mathfrak{g}_{\text {tot }}(M)$-module $\Lambda^{\bullet} V^{*}$ contains $H^{\bullet}(M, \mathbb{C})$ as a submodule. This gives the desired embedding of Hodge structures.

\section{$2.2 \quad F$-algebras}

Let $V$ be a vector space over an algebraically closed field of characteristic zero. Let $q$ be a non-degenerate scalar product on $V$, and $S^{\bullet} V$ the symmetric algebra. We will identify $V$ and $V^{*}$ via $q$. Then $q$ can be considered an element of $S^{2} V$. Multiplication by $q^{k}$ gives a natural embedding $S^{l} V \hookrightarrow$ $S^{l+2 k} V$. Denote by $R_{n, k}(V) \subset S^{n+k} V$ the orthogonal complement to the image of $S^{n-k} V \stackrel{q^{k}}{\rightarrow} S^{n+k} V$ with respect to the non-degenerate symmetric pairing on $S^{n+k} V$ induced by $q$. Let $F_{n}^{\bullet}(V)$ be the quotient of $S^{\bullet} V$ by the ideal generated by $\bigcup_{k} R_{n, k}(V)$, with the grading multiplied by two, so that $F_{n}^{2 i}(V)$ is the quotient of $S^{i} V$.

Definition 2.5: The algebra $F_{n}^{\bullet}(V)$ is called the $n$-th $F$-algebra of $V$. This is an even-graded algebra, with $\operatorname{dim} F^{4 n}(V)=1$.

Remark 2.6: By definition, the natural map $S^{i} V \longrightarrow F_{n}^{2 i}(V)$ is an isomorphism for $i \leqslant n$. For degree greater than $n$, one has $F_{n}^{2 n+2 i}(V) \cong S^{n-i} V$. It is clear from this description that $F_{n}^{2 n+2 i}(V)$ is dual to $F_{n}^{2 n-2 i}(V)$, that is, $F_{n}^{\bullet}(V)$ is a graded Frobenius algebra.

Remark 2.7: It is easy to see (see e. g. [Bo2]) that the $F$-algebra has the following description:

$$
\begin{gathered}
F_{n}^{\bullet}(V) \cong \frac{S^{\bullet} V}{\left\langle x^{n+1} \mid x \in V, q(x, x)=0\right\rangle} . \\
-7-
\end{gathered}
$$


N. Kurnosov, A. Soldatenkov, M. Verbitsky Kuga-Satake construction and cohomology of HK manifolds

Theorem 2.8: Let $F_{n}^{\bullet}(V)$ be the $F$-algebra of a vector space $V$, and $\mathfrak{g}$ the Lie algebra generated by all $\mathfrak{s l}(2)$-triples. Then $\mathfrak{g}$ is isomorphic to the Lie algebra $\mathfrak{g}=\mathfrak{s o}(\tilde{V})$, where $\tilde{V}=V_{0} \oplus V_{2} \oplus V_{4}$ is the Mukai extension of $(V, q)$ defined as in the Section 3.4. Moreover, $F_{n}^{\bullet}(V)$ is an irreducible g-representation generated by $1 \in F_{n}^{0}(V)$.

Proof: This is just an explicit description of irreducible components of the symmetric power of the fundamental representation of $\mathfrak{g}=\mathfrak{s o}(\tilde{V})$; see [V2] for more details.

\section{Clifford modules and the Kuga-Satake con- struction}

\subsection{Clifford algebras}

We start by fixing some notations involving Clifford algebras. For more details see $[\mathrm{LM}]$. In this section $\mathrm{k}$ will always denote a field of characteristic zero.

Let $H$ be a finite-dimensional vector space over $\mathrm{k}$ and $q \in S^{2} H^{*}$ a nondegenerate symmetric bilinear form. Let $T^{\bullet} H$ denote the tensor algebra and $I \subset T^{\bullet} H$ be the two-sided ideal generated by all elements of the form $v \otimes v-$ $q(v, v) \cdot 1$ for $v \in H$. The Clifford algebra is by definition $\mathcal{C}(H, q)=T^{\bullet} H / I$. When $H$ and $q$ are clear from the context, we will denote the Clifford algebra by $\mathcal{C}$. Recall that $\mathcal{C}$ is $\mathbb{Z} / 2 \mathbb{Z}$-graded $\mathcal{C}=\mathcal{C}^{0} \oplus \mathcal{C}^{1}$, and denote by $\alpha: \mathcal{C} \rightarrow \mathcal{C}$ the parity involution. Denote by $\beta: \mathcal{C} \rightarrow \mathcal{C}^{\text {op }}$ the antiautomorphism induced by $v_{1} \otimes \ldots \otimes v_{k} \mapsto v_{k} \otimes \ldots \otimes v_{1}$. We will use the notation $\bar{a}=\alpha \beta(a)$.

Recall that we have the canonical embedding $H \hookrightarrow \mathcal{C}$. The Clifford group is by definition $G=\left\{a \in \mathcal{C}^{\times} \mid \alpha(a) H a^{-1}=H\right\}$. The group $G$ comes with the natural action on $H$. Note that any non-isotropic $x \in H$ is contained in $G$. The action of $x$ on $H$ is by reflection: $v \mapsto-x v x^{-1}=v-2 q(x, x)^{-1} q(x, v) x$.

For any $a \in G$ and $v \in H$ we have $q\left(\alpha(a) v a^{-1}, \alpha(a) v a^{-1}\right)=\left(\alpha(a) v a^{-1}\right)^{2}=$ $a v^{2} a^{-1}=q(v, v)$, so the action of $G$ on $H$ is orthogonal, and we get a morphism $\rho: G \rightarrow \mathrm{O}(H, q)$. This morphism is surjective because $\mathrm{O}(H, q)$ is generated by reflections, and they are in the image of $\rho$. One can check that the kernel of $\rho$ consists of invertible scalars $\mathrm{k}^{\times}$([LM], Proposition 2.4).

Let $G^{i}=G \cap \mathcal{C}^{i}$. The surjectivity of $\rho$ and the description of its kernel imply that any element $a \in G$ is a product $a=x_{1} \cdot \ldots \cdot x_{n}$ where $x_{i} \in H$ are non-isotropic vectors. It follows that $G=G^{0} \coprod G^{1}$ and $G^{0}$ is a normal 
N. Kurnosov, A. Soldatenkov, M. Verbitsky Kuga-Satake construction and cohomology of HK manifolds

subgroup of index two. Moreover, if we define $N(a)=a \bar{a}$ then $N(a)=$ $(-1)^{n} \prod_{i=1}^{n} q\left(x_{i}, x_{i}\right) \in \mathrm{k}^{\times}$and $N(a)$ is called the norm of $a$. The group $\operatorname{Pin}(H, q)$ is by definition the kernel of $N$. We have $\operatorname{Pin}=\operatorname{Pin}^{0} \amalg \operatorname{Pin}^{1}$ and by definition $\operatorname{Spin}(H, q)=\operatorname{Pin}^{0}(H, q)$.

The Lie algebra $\mathfrak{s o}(H, q)$ can be canonically identified with $\Lambda^{2} H$ and embedded into $\mathcal{C}$ via the map $\Lambda^{2} H \rightarrow \mathcal{C}, x \wedge y \mapsto \frac{1}{2}(x y-y x)$. This identifies $\mathfrak{s o}(H, q)$ with the subspace of $\mathcal{C}$ spanned by the commutators of elements of $H$. The Lie bracket with any element of this subspace preserves $H$ and this gives the description of the canonical action of $\mathfrak{s o}(H, q)$ on $H$.

\subsection{Invariant forms on $\mathcal{C}$-modules}

Consider a $\mathbb{Z} / 2 \mathbb{Z}$-graded $\mathcal{C}$-module $V=V^{0} \oplus V^{1}$ with a bilinear form

$$
b: V \otimes V \rightarrow k
$$

Definition 3.1: The bilinear form $b$ is called $\mathcal{C}$-invariant if $b(a u, v)=b(u, \bar{a} v)$ for all $a \in \mathcal{C}$ and $u, v \in V$.

Remark 3.2: Our convention in this definition differs from what can be found in the literature by the parity automorphism. It makes no difference when we consider only the action of $\mathcal{C}^{0}$ on $V$, but in what follows it is important to use precisely the above definition.

If $b$ is $\mathcal{C}$-invariant, then for any $a \in G$ we have $b(a u, a v)=b(u, \bar{a} a v)=$ $N(\bar{a}) b(u, v)=N(a) b(u, v)$. In particular for $a \in \operatorname{Pin}(H, q)$ we have $N(a)=1$, and we see that $b \in\left(V^{*} \otimes V^{*}\right)^{\operatorname{Pin}(H, q)}$.

We will now consider $\mathcal{C}$ as a left module over itself. There exists a natural $\mathcal{C}$-invariant symmetric bilinear form on $\mathcal{C}$. For $u, v \in \mathcal{C}$ let

$$
\tau(u, v)=\operatorname{Tr}(u \bar{v})
$$

Here $\operatorname{Tr}(a)$ is the trace of $a$ considered as an endomorphism of $\mathcal{C}$. Note that the following relations hold: $\operatorname{Tr}(a)=\operatorname{Tr}(\alpha(a))=\operatorname{Tr}(\beta(a))=\operatorname{Tr}(\bar{a}) ; \operatorname{Tr}(u v)=$ $\operatorname{Tr}(v u) ; \operatorname{Tr}(a)=0$ for $a \in \mathcal{C}^{1}$. This clearly implies that $\tau(u, v)=\tau(v, u)$. Moreover, for any $a, u, v \in \mathcal{C}$ we have $\tau(a u, v)=\operatorname{Tr}(a u \bar{v})=\operatorname{Tr}(u \bar{v} a)=$ $\operatorname{Tr}(u \overline{\bar{a} v})=\tau(u, \bar{a} v)$, so the form $\tau$ is $\mathcal{C}$-invariant. The trace of an odd degree element is zero, so the form $\tau$ is even: $\tau \in S^{2}\left(\mathcal{C}^{0}\right)^{*} \oplus S^{2}\left(\mathcal{C}^{1}\right)^{*}$.

Definition 3.3: We will call the form $\tau$ described above the invariant trace form on $\mathcal{C}$. 
N. Kurnosov, A. Soldatenkov, M. Verbitsky Kuga-Satake construction and cohomology of HK manifolds

Lemma 3.4: Let $H$ be a quadratic vector space, $a \in H$ be a non-isotropic element, $H^{\prime} \subset H$ the orthogonal complement of $a$ and $\mathcal{C}^{\prime}=\mathcal{C}\left(H^{\prime}, q\right)$. Let $\tau^{\prime}$ be the corresponding trace form on $\mathcal{C}^{\prime}$. Then the Clifford algebra decomposes into $\tau$-orthogonal direct sum $\mathcal{C} \simeq \mathcal{C}^{\prime} \oplus a \mathcal{C}^{\prime}$. We have $\left.\tau\right|_{\mathcal{C}^{\prime}}=2 \tau^{\prime}$ and $\left.\tau\right|_{a \mathcal{C}^{\prime}}=$ $-2 q(a, a) \tau^{\prime}$

Proof: For $u, v \in \mathcal{C}^{\prime}$ we have $\tau(a u, v)=\operatorname{Tr}(a u \bar{v})$. The action of $a u \bar{v}$ on $\mathcal{C}^{\prime} \oplus a \mathcal{C}^{\prime}$ exchanges the summands, so the trace is zero and the decomposition $\mathcal{C} \simeq \mathcal{C}^{\prime} \oplus a \mathcal{C}^{\prime}$ is $\tau$-orthogonal.

Assume that $u, v \in \mathcal{C}^{\prime}$ have the same parity. In this case $a u \bar{v}=u \bar{v} a$ and the action of $u \bar{v}$ on $\mathcal{C}^{\prime} \oplus a \mathcal{C}^{\prime}$ is given by the matrix $\left(\begin{array}{cc}u \bar{v} & 0 \\ 0 & u \bar{v}\end{array}\right)$. We have $\tau(u, v)=\operatorname{Tr}(u \bar{v})=2 \operatorname{Tr}^{\prime}(u \bar{v})$ where $\operatorname{Tr}^{\prime}$ is the trace in $\mathcal{C}^{\prime}$. If $u$ and $v$ have different parity, the trace is zero. This proves that $\left.\tau\right|_{\mathcal{C}^{\prime}}=2 \tau^{\prime}$.

Note that $\tau(a u, a v)=\operatorname{Tr}(a u \bar{v} \bar{a})=-q(a, a) \operatorname{Tr}(u \bar{v})$. This implies that $\left.\tau\right|_{a \mathcal{C}^{\prime}}=-2 q(a, a) \tau^{\prime}$

Corollary 3.5: The invariant trace form $\tau$ is non-degenerate.

Proof: This follows from the previous lemma and induction on $\operatorname{dim} H$. For $\operatorname{dim} H=1$ we have $\mathcal{C} \simeq \mathrm{k}[x] /\left(x^{2}-d\right)$ for some $d \in \mathrm{k}^{\times}$and the trace form is clearly non-degenerate.

\subsection{The embedding}

Let $V$ be a $\mathcal{C}$-module of dimension $4 n$ with a $\mathcal{C}$-invariant symmetric bilinear form $\tau \in S^{2} V^{*}$. Assume that the form $\tau$ is non-degenerate. For $x \in H$ and $u, v \in V$ let $\omega_{x}(u, v)=\tau(x u, v)$. Since $\bar{x}=-x$, we have $\omega_{x}(u, v)=$ $-\tau(u, x v)=-\omega_{x}(v, u)$ and so $\omega_{x} \in \Lambda^{2} V^{*}$. We get a map

$$
\varphi: H \rightarrow \Lambda^{2} V^{*}, \quad x \mapsto \omega_{x}
$$

We will use the parity-twisted action of $\operatorname{Pin}(H, q)$ on $\Lambda^{2} V^{*}$ : for $\omega \in \Lambda^{2} V^{*}$, $a \in \operatorname{Pin}(H, q)$ and $u, v \in H$ let $(a \cdot \omega)(u, v)=\omega\left(a^{-1} u, \alpha(a)^{-1} v\right)$.

Lemma 3.6: In the above setting $\varphi$ is a morphism of $\operatorname{Pin}(H, q)$-modules. For any non-isotropic $x \in H$ the form $\omega_{x}$ is non-degenerate. 
Proof: For any $a \in \operatorname{Pin}(H, q)$ we have $\bar{a}=a^{-1}$. For any $x \in H$ and any $u, v \in V$ we obtain $\omega_{\alpha(a) x a^{-1}}(u, v)=\tau\left(\alpha(a) x a^{-1} u, v\right)=\tau\left(x a^{-1} u, \alpha(\bar{a}) v\right)=$ $\tau\left(x a^{-1} u, \alpha(a)^{-1} v\right)=\left(a \cdot \omega_{x}\right)(u, v)$, which proves the first claim.

The second claim follows from non-degeneracy of $\tau$ : for any $u \in V$ one can find $v \in V$ with $\tau(u, v) \neq 0$. Then $\omega_{x}(u, x v)=\tau(x u, x v)=-\tau\left(u, x^{2} v\right)=$ $-q(x, x) \tau(u, v)$ which is non-zero for non-isotropic $x$.

Denote by $W$ the image of $H$ in $\Lambda^{2} V^{*}$ under the map $\varphi$. Consider the polynomial $p \in S^{2 n} W^{*}$ given by $p(\omega)=\omega^{\wedge 2 n}$ (here we identify $\Lambda^{4 n} V^{*}$ with k). Analogously to [SV2, Lemma 3.10] we can prove that $W$ is a $k$-symplectic structure.

Lemma 3.7: In the above setting $p$ is proportional to $q^{n}$. For any isotropic $x \in H$ the form $\omega_{x}$ has rank $2 n$.

Proof: We may assume that the base field is algebraically closed. The polynomial $p$ is the restriction to $W$ of $P \in S^{2 n}\left(\Lambda^{2} V^{*}\right)$ defined in the same way: $P(\omega)=\omega^{\wedge 2 n}$. But $P$ is $\operatorname{Spin}(H, q)$-invariant since the action of $\operatorname{Spin}(H, q)$ on $\Lambda^{4 n} V^{*}$ is trivial $\left(\Lambda^{4 n} V^{*}\right.$ is one-dimensional). By Lemma 3.6 $H$ is embedded into $\Lambda^{2} V^{*}$ as a $\operatorname{Spin}(H, q)$-submodule, so $p$ is invariant under the $\operatorname{Spin}(H, q)$-action on $H$. This action factors through $\operatorname{SO}(H, q)$, so $p$ is invariant with respect to the special orthogonal group. Classical invariant theory (see $[\mathrm{CP}]$, Theorem 5.6) implies that $p$ is proportional to a power of the quadratic form $q$. We can choose the isomorphism $\Lambda^{4 n} V^{*} \simeq \mathrm{k}$ so that $p=q^{n}$.

Let $x \in H$ be isotropic and $\operatorname{dim}\left(\operatorname{ker} \omega_{x}\right)=2 r$. Pick a non-isotropic element $y \in H$ such that $q(x, y) \neq 0$. Consider the polynomial $\tilde{p}(t)=$ $p\left(\omega_{x}+t \omega_{y}\right)$. We have $\tilde{p}(t)=q(x+t y)^{n}$, so $\tilde{p}$ must have zero of order $n$ at $t=0$. But

$$
\begin{aligned}
\tilde{p}(t) & =\left(\omega_{x}+t \omega_{y}\right)^{\wedge 2 n}= \\
& =\left(\begin{array}{c}
2 n \\
r
\end{array}\right) t^{r} \omega_{x}^{\wedge(2 n-r)} \wedge \omega_{y}^{\wedge r}+\left(\begin{array}{c}
2 n \\
r+1
\end{array}\right) t^{r+1} \omega_{x}^{\wedge(2 n-r-1)} \wedge \omega_{y}^{\wedge(r+1)}+\ldots+t^{2 n} \omega_{y}^{\wedge 2 n},
\end{aligned}
$$

and $\omega_{x}^{\wedge(2 n-r)} \wedge \omega_{y}^{\wedge r} \neq 0$. So the order of zero at $t=0$ is $r$, hence $r=n$.

Let $A$ be the subalgebra in $\Lambda^{\bullet} V^{*}$ generated by $W \subset \Lambda^{2} V^{*}$. It is clear that $A$ is a quotient of the symmetric algebra $S^{\bullet} H$. We would like to describe the corresponding ideal in $S^{\bullet} H$.

Proposition 3.8: Assume that $\mathrm{k}=\overline{\mathrm{k}}$. Let $A^{\bullet}$ be the subalgebra in $\Lambda^{\bullet} V^{*}$ 
generated by $W$, where $V$ is a Clifford module over $\mathcal{C} \ell(H, q)$. Then $A^{\bullet}$ is isomorphic to the $F$-algebra $F_{n}^{\bullet}(H)$ (see Definition 2.5), where $n=\operatorname{dim}_{\mathrm{k}} V$.

Proof: We will identify $H$ and $H^{*}$ via $q$. The algebra $A$ is a quotient of $S^{\bullet} H$, let $I$ be the corresponding ideal. Note that $I$ contains all elements of the form $x^{n+1}$ for isotropic $x \in H$, as follows from Lemma 3.7.

Recall the following facts from representation theory of the orthogonal group $\mathrm{O}(H, q)$ (see $[\mathrm{How}])$. The form $q$ defines the trace maps $\operatorname{tr}: S^{k} H \rightarrow$ $S^{k-2} H$ whose kernels are called spaces of harmonic polynomials, we will denote them by $\mathcal{H}^{k}$. By definition $\mathcal{H}^{1}=H$. It is known that $\mathcal{H}^{k}$ are irreducible $\mathrm{O}(H, q)$-modules. We also have the following decomposition of $S^{k} H$ into irreducible $\mathrm{O}(H, q)$-modules:

$$
S^{k} H=\bigoplus_{r \geq 0} q^{r} \mathcal{H}^{k-2 r},
$$

where by $q$ we mean the element of $S^{2} H$, corresponding to the form $q$ under the isomorphism $H \simeq H^{*}$.

Note that for any $x \in H$ with $q(x, x)=0$ we have $\operatorname{tr}\left(x^{k}\right)=0$, hence $x^{k} \in \mathcal{H}^{k}$. Since $\mathcal{H}^{k}$ is irreducible, we see that it is spanned by the elements of the form $x^{k}$ with isotropic $x$. Also note that in the decomposition of the tensor product $\mathcal{H}^{k} \otimes \mathcal{H}^{1}$ into irreducible submodules we have two terms $\mathcal{H}^{k+1} \oplus \mathcal{H}^{k-1}$ plus some other components, not isomorphic to $\mathcal{H}^{m}$ (this can be checked using highest weight theory). So the image of the multiplication map $\mathcal{H}^{k} \otimes \mathcal{H}^{1} \rightarrow S^{k+1} H$ is contained in $\mathcal{H}^{k+1} \oplus q \mathcal{H}^{k-1}$. One can easily check that the image actually coincides with this direct sum. More generally, for any $k \geq m$ the image of the multiplication map $\mathcal{H}^{k} \otimes \mathcal{H}^{m} \rightarrow S^{k+m} H$ is $\mathcal{H}^{k+m} \oplus q \mathcal{H}^{k+m-2} \oplus \ldots \oplus q^{m} \mathcal{H}^{k-m}$.

Going back to our algebra $A^{\bullet}$, note that the ideal $I$ contains $\mathcal{H}^{n+1}$. Let us denote by $J$ the ideal generated by $\mathcal{H}^{n+1}$, then we have $J \subset I$ and $A$ is the quotient of $B^{\bullet}=S^{\bullet} V / J$. For $k \leq n$ we have $B^{k}=S^{k} H$. From the description of the multiplicative structure on irreducible components $\mathcal{H}^{m}$ it is easy to see that $B^{n+i} \simeq q^{i} B^{n-i}$ for all $i \geq 0$. In particular $B^{2 n} \simeq \mathrm{k}$.

We claim that multiplication maps $B^{k} \otimes B^{2 n-k} \rightarrow B^{2 n} \simeq \mathrm{k}$ give nondegenerate pairing on $B^{\bullet}$. In order to see this, take an element $x \in q^{r} \mathcal{H}^{k-2 r}$ for some $r$, so that $x=q^{r} \xi$ with $\xi \in \mathcal{H}^{k-2 r}$. Multiplication gives us surjective map $\mathcal{H}^{k-2 r} \otimes \mathcal{H}^{k-2 r} \rightarrow \mathcal{H}^{2 k-4 r} \oplus q \mathcal{H}^{2 k-4 r-2} \oplus \ldots \oplus q^{k-2 r} k$. Hence there exists an element $\eta \in \mathcal{H}^{k-2 r}$, such that $\xi \eta=q^{k-2 r}$. Then for $y=q^{n-k+r} \eta \in$ $q^{n-k+r} \mathcal{H}^{k-2 r} \subset B^{2 n-k}$ we have $x y=q^{n}$. This shows that the pairing is nondegenerate. In particular, all non-trivial ideals of the algebra $B^{\bullet}$ contain the element $q^{n}$ which generates $B^{2 n}$. 
N. Kurnosov, A. Soldatenkov, M. Verbitsky Kuga-Satake construction and cohomology of HK manifolds

Note that for the algebra $A^{\bullet}$ the graded component $A^{2 n}$ is non-zero, because $\left(\omega_{x}\right)^{\wedge 2 n} \neq 0$ for a non-isotropic $x \in H$. But if $A^{\bullet}$ were a quotient of $B^{\bullet}$ by some non-trivial ideal, this ideal would contain $B^{2 n} \simeq \mathrm{k}$ and $A^{2 n}$ would be trivial. We conclude that $A$ is isomorphic to $B^{\bullet}$.

\subsection{Mukai extension}

As above, let $(H, q)$ be a quadratic vector space. Let $\tilde{H}=\mathrm{k} \oplus H \oplus \mathrm{k}$ be the graded vector space with direct summands of degree 0, 2 and 4. Define the quadratic form $\tilde{q}$ on $\tilde{H}$ : let $\tilde{q}\left((a, x, b),\left(a^{\prime}, x^{\prime}, b^{\prime}\right)\right)=q\left(x, x^{\prime}\right)-a b^{\prime}-a^{\prime} b$, so that degree 0 and degree 4 summands make up a hyperbolic plane which is orthogonal to $H$, and the restriction of $\tilde{q}$ to $H$ is $q$. We will refer to $(\tilde{H}, \tilde{q})$ as Mukai extension of $(H, q)$. The Lie algebra $\mathfrak{g}=\mathfrak{s o}(\tilde{H}, \tilde{q})$ has the induced grading $\mathfrak{g}=\mathfrak{g}_{-2} \oplus \mathfrak{g}_{0} \oplus \mathfrak{g}_{2}$. Its components can be described as follows.

Lemma 3.9: We have $\mathfrak{g}_{2} \simeq \mathfrak{g}_{-2} \simeq H$ as vector spaces, $\mathfrak{g}_{0} \simeq \mathrm{k} \oplus \mathfrak{s o}(H, q)$ as subalgebra (the first summand is the center of $\mathfrak{g}_{0}$ ). The action of $\mathfrak{g}_{0}$ on $\mathfrak{g}_{-2}$ and $\mathfrak{g}_{2}$ is via the standard representation of $\mathfrak{s o}(H, q)$. The Lie bracket of two elements $x \in \mathfrak{g}_{-2}$ and $y \in \mathfrak{g}_{2}$ is given by $[x, y]=(q(x, y), x \wedge y) \in \mathfrak{g}_{0}$, where we use the natural isomorphism $\mathfrak{s o}(H, q) \simeq \Lambda^{2} H$.

Proof: Any element $x \in H$ defines two endomorphisms of $\tilde{H}$ of degree 2 and -2 : one is given by $(a, z, b) \mapsto(0, a x, q(x, z))$, the other by $(a, z, b) \mapsto$ $(q(x, z), b x, 0)$. It is straightforward to check that these endomorphisms are contained in $\mathfrak{g}$. It is also easy to check the stated commutator relations. Hence we get two embeddings $H \hookrightarrow \mathfrak{g}_{-2}$ and $H \hookrightarrow \mathfrak{g}_{2}$. The generator of the center of $\mathfrak{g}_{0}$ acts as $(a, z, b) \mapsto(-2 a, 0,2 b)$. The embedding $\mathfrak{s o}(H, q) \hookrightarrow \mathfrak{g}_{0}$ is the obvious one. All these embeddings are isomorphisms for dimension reasons.

\subsection{The Lie algebra action}

Recall that for a quadratic vector space $(H, q)$ we denote by $\mathcal{C}$ the Clifford algebra $\mathcal{C}(H, q)$. For any $\mathcal{C}$-module $V$ with a non-degenerate $\mathcal{C}$-invariant symmetric bilinear form $\tau \in S^{2} V^{*}$ we have constructed the embedding $\varphi: H \rightarrow \Lambda^{2} V^{*}$ (see 3.1$)$.

We will show that the embedding $\varphi$ induces an action of the Mukaiextended Lie algebra $\mathfrak{s o}(\tilde{H}, \tilde{q})$ on $\Lambda^{\bullet} V^{*}$ compatible with the grading.

For $x \in H$ we will denote its image in $\Lambda^{2} V^{*}$ by $\omega_{x}$. Consider the endomorphisms $\mathrm{L}_{x}, \Lambda_{x} \in \operatorname{End}\left(\Lambda^{\bullet} V^{*}\right)$ given by $\left.\mathrm{L}_{x} \eta=\omega_{x} \wedge \eta, \Lambda_{x} \eta=\omega_{x}\right\lrcorner \eta$ (to 
define the convolution with $\omega_{x}$ we identify $V$ and $V^{*}$ via $\tau$ ). The action of $a \in \mathcal{C}$ on $V$ can be extended to a derivation of the algebra $\Lambda^{\bullet} V^{*}$ which we will denote by $\delta_{a}$. Denote by $\Xi$ the endomorphism of $\Lambda^{\bullet} V^{*}$ that acts on the homogeneous component of degree $k$ as multiplication by $k-\frac{1}{2} \operatorname{dim} V$.

Theorem 3.10: The Lie subalgebra of $\operatorname{End}\left(\Lambda^{\bullet} V^{*}\right)$ generated by $\mathrm{L}_{x}, \Lambda_{x}$ for all $x \in H$ is isomorphic to the orthogonal Lie algebra $\mathfrak{s o}(\tilde{H}, \tilde{q})$ of the Mukai extension $\tilde{H}$.

Proof: Recall that $\mathfrak{s o}(H, q) \simeq \Lambda^{2} H \hookrightarrow \mathcal{C}, x \wedge y \mapsto \frac{1}{2}(x y-y x)$ is an isomorphism of $\mathfrak{s o}(H, q)$ and a Lie subalgebra of $\mathcal{C}$ spanned by commutators of elements of $H$. Hence the operators $\delta_{\frac{1}{2}(x y-y x)}$ span a subalgebra isomorphic to $\mathfrak{s o}(H, q)$. The statement of the theorem now follows from Lemma 3.9 and Lemma 3.12 by identifying $\mathfrak{g}_{2}$ with the subspace spanned by all $\mathrm{L}_{x}$ for $x \in H$, $\mathfrak{g}_{-2}$ with the subspace spanned by all $\Lambda_{x}$ and the unit element of the center of $\mathfrak{g}_{0}$ with $\Xi$.

Remark 3.11: In the case $\mathrm{k}=\mathbb{R}$ and $(H, q)$ the cohomology of a hyperkähler manifold with BBF form, one can prove the above theorem by checking that the $\mathfrak{s o}(4,1)$-relations hold for any non-degenerate 3-dimensional subspace $H_{3} \subset H$.

Lemma 3.12: For any $x, y \in H$ we have

$$
\left[\Lambda_{x}, \mathrm{~L}_{y}\right]=\delta_{\frac{1}{2}(x y-y x)}+q(x, y) \Xi .
$$

Proof: Below we will give an explicit argument, based on computations. However, these computations are possible to avoid as follows: first, one checks (3.2) in a 3-symplectic space (this is true and well known because for 3-symplectic space the algebra generated by $L_{\omega_{i}}$ and $\Lambda_{\omega_{j}}$ is known and described explicitly in Appendix (Remark A.7). Then, one can notice that it is possible to find a 3-symplectic structure on $V$ containing any generic $x, y \in H \subset \Lambda^{2} V^{*}$, and hence the relation (3.2) is true on a Zariski dense set of $x, y$.

We can assume that $\mathrm{k}=\overline{\mathrm{k}}$. Since non-isotropic vectors form a Zariskiopen subset of $H$, it is enough to prove the identity assuming that $x$ is non-isotropic. So we fix a pair of elements $x, y \in H$ with $q(x, x) \neq 0$. Note that for any $u \in V$ we have $\tau(x u, u)=0$, so we can find a basis $V=$ $\left\langle e_{1}, f_{1}, \ldots, e_{2 n}, f_{2 n}\right\rangle$ where $f_{i}=x e_{i}$ and all elements of the basis are pairwise orthogonal. We can also assume that $\tau\left(e_{i}, e_{i}\right)=1$ and then $\tau\left(f_{i}, f_{i}\right)=$ 
$-q(x, x)$. In the dual basis $\omega_{x}=-q(x, x) \sum e_{i}^{*} \wedge f_{i}^{*}$. Using $\tau$ to identify $V$ and $V^{*}$ we can write $\omega_{x}=\sum e_{i} \wedge f_{i}$.

Let $\eta \in \Lambda^{k} V^{*}$ and $\xi_{1}, \ldots, \xi_{k} \in V$. We have

$$
\begin{aligned}
& \left(\Lambda_{x} \mathrm{~L}_{y} \eta\right)\left(\xi_{1}, \ldots, \xi_{k}\right)=\sum_{p=1}^{2 n}\left(\omega_{y} \wedge \eta\right)\left(e_{p}, f_{p}, \xi_{1}, \ldots, \xi_{k}\right)= \\
& \sum_{p=1}^{2 n} \tau\left(y e_{p}, f_{p}\right) \eta\left(\xi_{1}, \ldots, \xi_{k}\right)+\sum_{p=1}^{2 n} \sum_{i=1}^{k}(-1)^{i} \tau\left(y e_{p}, \xi_{i}\right) \eta\left(f_{p}, \ldots, \check{\xi}_{i}, \ldots\right)+ \\
& \sum_{p=1}^{2 n} \sum_{i=1}^{k}(-1)^{i-1} \tau\left(y f_{p}, \xi_{i}\right) \eta\left(e_{p}, \ldots, \check{\xi}_{i}, \ldots\right)+ \\
& \sum_{p=1}^{2 n} \sum_{i<j}(-1)^{i+j-1} \tau\left(y \xi_{i}, \xi_{j}\right) \eta\left(e_{p}, f_{p}, \ldots, \check{\xi}_{i}, \ldots, \check{\xi}_{j}, \ldots\right) .
\end{aligned}
$$

The last term in this formula is equal to $\left(\mathrm{L}_{y} \Lambda_{x} \eta\right)\left(\xi_{1}, \ldots, \xi_{k}\right)$. The sum of the second and third terms is $\left(\delta_{x y} \eta\right)\left(\xi_{1}, \ldots, \xi_{k}\right)$, where we use that

$$
\xi_{i}=\sum_{j}\left(\tau\left(\xi_{i}, e_{j}\right) e_{j}-\frac{1}{q(x, x)} \tau\left(\xi_{i}, f_{j}\right) f_{j}\right) .
$$

The sum $\sum_{p} \tau\left(y e_{p}, f_{p}\right)=\sum_{p} \tau\left(y e_{p}, x e_{p}\right)$ can be rewritten in terms of the trace of $x y$ acting on $V$ : we have $\operatorname{Tr}(x y)=\sum_{i}\left(e_{i}^{*}\left(x y e_{i}\right)+f_{i}^{*}\left(x y f_{i}\right)\right)=$ $\sum_{i}\left(\tau\left(e_{i}, x y e_{i}\right)-q(x, x)^{-1} \tau\left(f_{i}, x y f_{i}\right)\right)=-2 \sum_{i} \tau\left(x e_{i}, y e_{i}\right)$. We have obtained the following formula:

$$
\left[\Lambda_{x}, \mathrm{~L}_{y}\right]=\delta_{x y}-\frac{1}{2} \operatorname{Tr}(x y) \mathrm{Id} .
$$

Using the identity $x y=\frac{1}{2}(x y-y x)+q(x, y)$ we see that $\operatorname{Tr}(x y)=q(x, y) \operatorname{dim} V$, and since $\delta_{q(x, y)} \eta=k q(x, y) \eta$ we have finished the proof.

Corollary 3.13: For any non-isotropic $x \in H$ the operators $\mathrm{L}_{x}, \Xi$ and $-q(x, x)^{-1} \Lambda_{x}$ form an $\mathfrak{s l}_{2}$-triple in $\operatorname{End}\left(\Lambda^{\bullet} V^{*}\right)$.

Proof: The identity $\left[\mathrm{L}_{x},-q(x, x)^{-1} \Lambda_{x}\right]=\Xi$ follows from the lemma. The identities $\left[\Xi, \mathrm{L}_{x}\right]=2 \mathrm{~L}_{x}$ and $\left[\Xi,-q(x, x)^{-1} \Lambda\right]=-2\left(-q(x, x)^{-1} \Lambda_{x}\right)$ follow from the fact that $\mathrm{L}_{x}$ is of degree 2 and $\Lambda_{x}$ is of degree -2 .

We obtain an action of the Lie algebra $\mathfrak{s o}(\tilde{H}, \tilde{q})$ on $\Lambda^{\bullet} V^{*}$ for any $\mathcal{C}$-module $V$ with an invariant symmetric bilinear form $\tau$. Consider a pair of such modules $V_{1}, V_{2}$ with the forms $\tau_{1}, \tau_{2}$ and let $\varphi_{i}: H \rightarrow \Lambda^{2} V_{i}^{*}$ be two embeddings 
constructed as above. On $V=V_{1} \oplus V_{2}$ we have the form $\tau_{1} \oplus \tau_{2}$ and it is clear by construction that $\varphi: H \rightarrow \Lambda^{2} V^{*}$ equals the composition of $\left(\varphi_{1}, \varphi_{2}\right): H \rightarrow$ $\Lambda^{2} V_{1}^{*} \oplus \Lambda^{2} V_{2}^{*}$ and the natural embedding $\Lambda^{2} V_{1}^{*} \oplus \Lambda^{2} V_{2}^{*} \hookrightarrow \Lambda^{2} V^{*}$. This implies that the natural isomorphism $\Lambda^{\bullet}\left(V_{1} \oplus V_{2}\right)^{*} \simeq \Lambda^{\bullet} V_{1}^{*} \otimes \Lambda^{\bullet} V_{2}^{*}$ is compatible with the action of $\mathfrak{s o}(\tilde{H}, \tilde{q})$.

Theorem 3.14: For any quadratic vector space $(H, q)$ and any representation $W$ of $\mathfrak{g}=\mathfrak{s o}(\tilde{H}, \tilde{q})$ there exists a $\mathcal{C}(H, q)$-module $V$ with invariant symmetric bilinear form $\tau$, such that $\Lambda^{\bullet} V^{*}$ contains $W$ as a $\mathfrak{g}$-submodule.

Proof: Step 1: Assume additionally that the representation $W$ is irreducible. We denote $\mathcal{C}=\mathcal{C}(H, q)$. Let $V_{1}$ denote $\mathcal{C}$ as the left $\mathcal{C}$-module with $\tau_{1}$ the invariant trace form. The action of $\mathfrak{s o}(\tilde{H}, \tilde{q})$ on $\Lambda^{\bullet} V_{1}^{*}$ induces an action of the group $\operatorname{Spin}(\tilde{H}, \tilde{q})$. Let us prove that this action is faithful. The construction behaves naturally under the base field extensions, so we may assume that $\mathrm{k}=\overline{\mathrm{k}}$. The Spin-group is connected, and we have to check that its center acts non-trivially on $\Lambda^{\bullet} V_{1}^{*}$.

The structure of the center of Spin depends on $\operatorname{dim}(H)$ modulo 4. If the dimension is odd, then the only non-trivial element of the center is $-1 \in \operatorname{Spin}(\tilde{H}, \tilde{q}) \subset \mathcal{C} \ell(\tilde{H}, \tilde{q})$. This element is also contained in $\operatorname{Spin}(H, q) \subset$ $\operatorname{Spin}(\tilde{H}, \tilde{q})$ and it clearly acts non-trivially on $V_{1}^{*} \subset \Lambda^{\bullet} V_{1}^{*}$. In the case when the dimension of $H$ is even, we have to consider one more element of the center. It can be described as follows. Let $\tilde{H}=\left\langle e_{1}, \ldots, e_{2 d}, f_{1}, f_{2}\right\rangle$ be the orthonormal basis, such that the elements $f_{1}$ and $f_{2}$ span the orthogonal complement to $H \subset \tilde{H}$. Then the central element is $g=e_{1} \ldots e_{2 d} f_{1} f_{2}$. Observe that $g$ is the product of $g_{1}=e_{1} \ldots e_{2 d} \in \operatorname{Spin}(H, q)$ and $g_{2}=f_{1} f_{2} \in \operatorname{Spin}(U)$ where $U$ is the hyperbolic plane. Under the isomorphism $\operatorname{Spin}(U) \simeq k^{\times}$the element $g_{2}$ corresponds to $\sqrt{-1}$. The action of $g_{1}$ on $\mathbb{P}\left(V_{1}^{*}\right)$ is non-trivial and the action of $g_{2}$ is trivial. Hence the action of $g$ on $V_{1}^{*}$ is non-trivial.

Since $\Lambda^{\bullet} V_{1}^{*}$ is a faithful $\operatorname{Spin}(\tilde{H}, \tilde{q})$-module, by [DMOS], Proposition 3.1 (p. 40), any irreducible representation of $\operatorname{Spin}(\tilde{H}, \tilde{q})$ is contained in $\left(\Lambda^{\bullet} V_{1}^{*}\right)^{\otimes N}$ for $N$ big enough. Then we can take $V=V_{1}^{\oplus N}$.

Step 2: Consider the case when $W$ is not necessarily irreducible. We will prove that $W$ can still be embedded into $\left(\Lambda^{\bullet} V_{1}^{*}\right)^{\otimes N}$ if $N$ is big enough. We use induction on the number of irreducible components of $W$. Let $W \simeq W^{\prime} \oplus W^{\prime \prime}$, where $W^{\prime}$ is irreducible.

Let us denote by $L$ the trivial one-dimensional representation of $\operatorname{Spin}(\tilde{H}, \tilde{q})$. There exists $k$, such that the representation $U=\left(\Lambda^{\bullet} V_{1}^{*}\right)^{\otimes k}$ contains $L$, so that $U \simeq U^{\prime} \oplus L$ for some representation $U^{\prime}$. On the other hand, $U \simeq \Lambda^{\bullet}\left(\left(V_{1}^{*}\right)^{\oplus k}\right)$ 
is still a faithful $\operatorname{Spin}(\tilde{H}, \tilde{q})$-module by the argument from step 1 . This implies that $U^{\prime}$ is faithful. It follows that for big $N^{\prime}$ the representation $U^{\otimes N^{\prime}} \simeq\left(\Lambda^{\bullet} V_{1}^{*}\right)^{\otimes k N^{\prime}}$ contains both the irreducible representation $W^{\prime}$ and $L$. By induction, we can assume that $U^{\otimes N^{\prime \prime}}$ contains both $W^{\prime \prime}$ and $L$, for some $N^{\prime \prime}$. Then $U^{\otimes N^{\prime}} \otimes U^{\otimes N^{\prime \prime}} \simeq\left(\Lambda^{\bullet} V_{1}^{*}\right)^{\otimes k\left(N^{\prime}+N^{\prime \prime}\right)}$ contains $W^{\prime} \oplus W^{\prime \prime} \simeq W$ and $L$. This completes the induction step.

\section{Multidimensional Kuga-Satake construction}

\subsection{The embedding}

Let us apply the results of Section 3 to the cohomology of hyperkähler manifolds. This will give a multidimensional analogue of the Kuga-Satake construction.

Theorem 4.1: Let $M$ be a hyperkähler manifold. There exists an integer $l \geq 0$, a complex torus $T$, an embedding $\mathfrak{g}_{\text {tot }}(M) \hookrightarrow \mathfrak{g}_{\text {tot }}(T)$ of Lie algebras, and an embedding $\Psi: H^{\bullet}(M, \mathbb{C}) \hookrightarrow H^{\bullet+l}(T, \mathbb{C})$ of $\mathfrak{g}_{\text {tot }}(M)$-modules. For each complex structure $I$ of hyperkähler type on $M$ there exists a complex structure on $T$ such that $\Psi$ is a morphism of Hodge structures.

Proof: Let $H=H^{2}(M, \mathbb{R})$ and $q$ be the BBF form (see Definition 1.8). We know that $\mathfrak{g}_{\text {tot }}(M)=\mathfrak{s o}(\tilde{H}, \tilde{q})$ (see Theorem A.10). We apply the construction from Section 3.3 and Theorem 3.14 to the pair $(H, q)$ and to $W=$ $H^{\bullet}(M, \mathbb{R})$. We obtain a $\mathcal{C}(H, q)$-module $V$, an embedding $H \hookrightarrow \Lambda^{2} V^{*}$ (a $k$-symplectic structure on $V$ ) and an embedding $W \hookrightarrow \Lambda^{\bullet} V^{*}$ of $\mathfrak{g}_{\text {tot }}(M)$ modules. Note that the grading on $W$ and $\Lambda^{\bullet} V^{*}$ is induced by the action of $\mathfrak{g}_{\text {tot }}(M)$, so we have a degree $l$ morphism $\Psi: H^{\bullet}(M, \mathbb{R}) \hookrightarrow \Lambda^{\bullet+l} V^{*}$ of graded vector spaces, for some $l$ (the shift of degree is due to the difference of dimensions of $M$ and $V$ ). We define $T$ to be the quotient of $V$ by a lattice. Note that both $V$ and the morphism $\Psi$ are defined over $\mathbb{Q}$, since the BBF form is defined over $\mathbb{Q}$, and the constructions from Section 3 behave naturally under base change.

The Hodge decomposition on $H^{\bullet}(M, \mathbb{C})$ is given by the $\mathfrak{u}(1)$-action which belongs to the Lie algebra $\mathfrak{s o}(H, q) \subset \mathfrak{s o}(\tilde{H}, \tilde{q})$ (see Section A.2). The corresponding element of $\mathfrak{s o}(H, q)$ is a skew-symmetric matrix $\mu$ of rank two. Let $P=\left\langle e_{1}, e_{2}\right\rangle$, where $e_{1}=\operatorname{Re} \Theta, e_{2}=\operatorname{Im} \Theta$ and $\Theta$ is a generator of $H^{2,0}(M)$, normalized so that $q\left(e_{i}, e_{i}\right)=1$. Then $\mu=e_{1} e_{2} \in \mathfrak{s o}(H, q) \subset \mathcal{C} \ell(H, q)$. It acts trivially on the orthogonal complement to the 2-dimensional subspace 
$P$, and as $\left(\begin{array}{cc}0 & 2 \\ -2 & 0\end{array}\right)$ on $P$. However, on each Clifford module $\mu$ acts as a complex structure, because $\mu^{2}=-1$ in the Clifford algebra. Therefore, $\mu$ acts as a complex structure on $H^{1}(T, \mathbb{R})$.

The weight decomposition under the $e^{t \mu}$-action on $H^{\bullet}(M, \mathbb{C})$ and $H^{\bullet}(T, \mathbb{C})$ gives the Hodge decomposition on these cohomology spaces, hence $\Psi$ is compatible with the Hodge structures.

\subsection{Polarization}

Like in the classical Kuga-Satake construction, in the case when the hyperkähler manifold $M$ admits a polarization, the complex torus $T$ from Theorem 4.1 admits a polarization too. The proof of this is essentially the same as in the classical case.

Let $H_{\mathbb{Q}}=H^{2}(M, \mathbb{Q}), H=H_{\mathbb{Q}} \otimes \mathbb{R}, h \in H_{\mathbb{Q}}$ an ample class and $H^{\prime} \subset H$ the orthogonal complement of $h$ with respect to the BBF form $q$. Recall from the proof of Theorem 4.1 that $T$ is a quotient of $V=V_{1}^{\oplus N}$ for sufficiently big $N$, where $V_{1} \simeq \mathcal{C}(H, q)$. To prove that $T$ is polarized it is enough to construct a rational Hermitian form $\sigma \in \Lambda^{2} V_{1}^{*}$. Let $\mathcal{C}^{\prime}=\mathcal{C}\left(H^{\prime},\left.q\right|_{H^{\prime}}\right)$. Note that $\mathcal{C} \ell(H, q) \simeq \mathcal{C}^{\prime} \oplus h \mathcal{C}^{\prime}$ and this induces the decomposition $V_{1} \simeq \mathcal{C}^{\prime \oplus 2}$ of rational Hodge structures, because the Hodge structure on $V_{1}$ is defined by a Weil operator which lies in $\mathcal{C}^{\prime}$. Hence it is enough to produce a polarization on $\mathcal{C}^{\prime}$.

We have reduced the problem to the following: $\left(H_{\mathbb{Q}}, q\right)$ is a rational quadratic vector space, $q$ has signature $(2, n)$ for some $n$ and we need to construct a polarization on $V=\mathcal{C} \ell(H, q)$ where $H=H_{\mathbb{Q}} \otimes \mathbb{R}$. Let $e_{1}, e_{2} \in H_{\mathbb{Q}}$ span a two-dimensional subspace in $H$ on which $q$ is positive. Assume that $q\left(e_{1}, e_{2}\right)=0$ and denote $a=e_{1} e_{2} \in \mathcal{C} \ell\left(H_{\mathbb{Q}}, q\right)$. For $x, y \in \mathcal{C} \ell(H, q)$ let

$$
\sigma_{a}(x, y)=\operatorname{Tr}(x a \bar{y}) .
$$

Proposition 4.2: Either $\sigma_{a}$ or $-\sigma_{a}$ defines a polarization on $V$.

Proof: It is easy to check that $\sigma_{a}$ is skew-symmetric, non-degenerate and $\operatorname{Pin}(H, q)$-invariant. The complex structure on $V$ is given by an element $\mu=e_{1}^{\prime} e_{2}^{\prime} \in \mathfrak{s o}(H, q) \subset \mathcal{C} \ell(H, q)$, where $e_{i}^{\prime} \in H$ are such that $q\left(e_{i}^{\prime}, e_{i}^{\prime}\right)=1$, $q\left(e_{1}^{\prime}, e_{2}^{\prime}\right)=0$. We need to check that $\sigma_{a}(x, \mu x) \neq 0$ for $x \neq 0$, because this would imply that $\sigma_{a}$ is sign-definite.

Note that we can find an element $g \in \operatorname{Spin}^{+}(H, q)$ such that $\mu$ is proportional to $\mathrm{gag}^{-1}$. Here $\operatorname{Spin}^{+}(H, q)$ is the connected component of the identity 
N. Kurnosov, A. Soldatenkov, M. Verbitsky Kuga-Satake construction and cohomology of HK manifolds

in the Spin-group. The orbit of $\sigma_{a}$ under the action of $\operatorname{Spin}^{+}(H, q)$ is connected, and all forms in this orbit are non-degenerate. Hence the form $\sigma_{a}$ is sign-definite if and only if $\sigma_{\mu}$ is. So it is enough to prove that $\sigma_{\mu}(x, \mu x) \neq 0$ for $x \neq 0$.

We prove this by induction on $\operatorname{dim} H$. Consider the orthogonal decomposition $H=P \oplus H^{\prime}$, where $P=\left\langle e_{1}^{\prime}, e_{2}^{\prime}\right\rangle$. Pick a non-zero element $z \in H^{\prime}$ and let $H^{\prime \prime}$ be the orthogonal complement to $z$. Let $\mathcal{C}^{\prime \prime}=\mathcal{C} \ell\left(H^{\prime \prime},\left.q\right|_{H^{\prime \prime}}\right)$, then $V \simeq \mathcal{C}^{\prime \prime} \oplus z \mathcal{C}^{\prime \prime}$. One can check like in the proof of Lemma 3.4 that $\left.\sigma_{\mu}\right|_{\mathcal{C}^{\prime \prime}}=2 \sigma_{\mu}^{\prime \prime}$ and $\left.\sigma_{\mu}\right|_{z \mathcal{C}^{\prime \prime}}=-2 q(z, z) \sigma_{\mu}^{\prime \prime}$, where $\sigma_{\mu}^{\prime \prime}$ is the corresponding form on $\mathcal{C}^{\prime \prime}$. Since $q(z, z)<0$, it is enough to prove that $\sigma_{\mu}^{\prime \prime}$ is positive-definite, and so we can replace $H$ by $H^{\prime \prime}$. This completes the induction step. It remains to consider the case when $H=P$. In this case $\mathcal{C}(H, q)$ is the algebra of $2 \times 2$ real matrices. One can check that $\sigma_{\mu}$ is positive definite on this algebra by a straightforward computation.

Remark 4.3: The form $\sigma_{a}$ which we use to define the polarization is not contained in the image of the embedding $\varphi$ from 3.1.

\section{A Supersymmetry in hyperkähler geometry}

We give a summary of results which are used to study the cohomology of a hyperkähler manifold. We follow [V3] and [V4]; see also [FKS].

\section{A.1 Supersymmetry on Kähler manifolds}

Let $(M, I, g)$ be a compact Kähler manifold, $\omega$ its Kähler form, and $\Lambda^{\bullet}(M)$ its de Rham algebra. On $\Lambda^{\bullet}(M)$, the following operators are defined:

1. de Rham differential $d$, its adjoint $d^{*}$ and the Laplacian $\Delta$;

2. the Lefschetz operator $\mathrm{L}(\alpha)=\omega \wedge \alpha$ and its adjoint $\Lambda(\alpha):=* \mathrm{~L} * \alpha$;

3. the Weil operator $\left.W\right|_{\Lambda^{p, q(M)}}=\sqrt{-1}(p-q)$.

The following theorem is a convenient way to summarize the Kähler relations and the Lefschetz $\mathfrak{s l}(2)$-action. 
N. Kurnosov, A. Soldatenkov, M. Verbitsky Kuga-Satake construction and cohomology of HK manifolds

Theorem A.1: These operators generate a Lie superalgebra $\mathfrak{a}$ of dimension $(5 \mid 4)$, acting on $\Lambda^{\bullet}(M)$. Moreover, the Laplacian $\Delta$ is central in $\mathfrak{a}$, hence $\mathfrak{a}$ also acts on the cohomology of $M$.

Corollary A.2: The $\mathfrak{s l}(2)$-action of $\langle\mathrm{L}, \Lambda, \Xi\rangle$ and the action of the Weil operator $W$ commute with Laplacian, hence preserve the harmonic forms on a Kähler manifold.

\section{A.2 Supersymmetry on hyperkähler manifolds}

Let $(M, I, J, K, g)$ be a hyperkähler manifold, $\omega_{I}, \omega_{J}, \omega_{K}$ its Kähler forms. On $\Lambda^{\bullet}(M)$, the following operators are defined:

1. de Rham differential $d$, its adjoint $d^{*}$ and the Laplacian $\Delta$;

2. The Lefschetz operators

$$
\mathrm{L}_{I}(\alpha)=\omega_{I} \wedge \alpha, \quad \mathrm{L}_{J}(\alpha)=\omega_{J} \wedge \alpha, \quad \mathrm{L}_{K}(\alpha)=\omega_{K} \wedge \alpha
$$

and their adjoints

$$
\Lambda_{I}(\alpha)=* \mathrm{~L}_{I} * \alpha, \quad \Lambda_{J}(\alpha)=* \mathrm{~L}_{J} * \alpha, \quad \Lambda_{K}(\alpha)=* \mathrm{~L}_{K} * \alpha
$$

3. The Weil operators $\left.W_{I}\right|_{\Lambda^{p, q(M, I)}}=\sqrt{-1}(p-q),\left.W_{J}\right|_{\Lambda^{p, q}(M, J)}=\sqrt{-1}(p-$ $q),\left.W_{K}\right|_{\Lambda^{p, q}(M, K)}=\sqrt{-1}(p-q)$.

The following result is an analogue of Theorem A.1:

Theorem A.3: These operators generate a Lie superalgebra $\mathfrak{a}$ of dimension (11|8), acting on $\Lambda^{\bullet}(M)$. Moreover, the Laplacian $\Delta$ is central in $\mathfrak{a}$, hence $\mathfrak{a}$ also acts on the cohomology of $M$.

Remark A.4: The Weil operators span the Lie algebra $\mathfrak{s u}(2)$ of unitary quaternions. In fact, one has $\left[\mathrm{L}_{I}, \Lambda_{J}\right]=W_{K},\left[\mathrm{~L}_{J}, \Lambda_{K}\right]=W_{I},\left[\mathrm{~L}_{I}, \Lambda_{K}\right]=$ $-W_{J}$ (see $[\mathrm{V} 0]$ ). This means that the quaternionic action belongs to $\mathfrak{a}$. The twisted de Rham differentials $d_{I}, d_{J}, d_{K}$, associated to $I, J, K$ also belong to $\mathfrak{a}: d_{I}=\left[W_{I}, d\right], d_{J}=\left[W_{J}, d\right], d_{K}=\left[W_{K}, d\right]$. 
N. Kurnosov, A. Soldatenkov, M. Verbitsky Kuga-Satake construction and cohomology of HK manifolds

Remark A.5: The action of $\mathfrak{a}_{\text {even }}$ on $\mathfrak{a}_{\text {odd }}$ is the fundamental representation of $\mathfrak{s p}(1,1, \mathbb{H})$ in $\mathbb{H}^{2}$, with the quaternionic Hermitian metric on $\mathfrak{a}_{\text {odd }}$ provided by the anticommutator. This implies that the odd part

$$
\left\langle d, d_{I}, d_{J}, d_{K}, d^{*}, d_{I}^{*}, d_{J}^{*}, d_{K}^{*}\right\rangle
$$

generates the 9-dimensional odd Heisenberg algebra, with the only non-trivial supercommutators being

$$
\left\{d, d^{*}\right\}=\left\{d_{I}, d_{I}^{*}\right\}=\left\{d_{J}, d_{J}^{*}\right\}=\left\{d_{K}, d_{K}^{*}\right\}=\Delta .
$$

Remark A.6: The even part of $\mathfrak{a}$ is isomorphic to $\mathfrak{s p}(1,1, \mathbb{H}) \oplus \mathbb{R} \cdot \Delta$. The natural homomorphism to $\mathfrak{s p}(1,1, \mathbb{H}) \oplus \mathbb{R} \cdot \Delta$ is given by the previous remark. To see that it is an isomorphism we use the dimension count.

Remark A.7: The algebra $\mathfrak{s p}(1,1, \mathbb{H})$ is isomorphic to $\mathfrak{s o}(4,1)$. To construct this isomorphism, we consider the action of $\mathfrak{a}$ on the 5 -dimensional graded vector space $H^{\bullet}=H^{0} \oplus H^{2} \oplus H^{4}$, with $H^{0}=\mathbb{R}, H^{2}=\left\langle\omega_{I}, \omega_{J}, \omega_{K}\right\rangle, H^{4}=\mathbb{R}$, defined in the same way as the action of $\mathfrak{a}$ on the 5 -dimensional subspace in the cohomology of a hyperkähler manifold of real dimension 4 .

Remark A.8: A Cartan subalgebra of

$$
\mathfrak{s o}(4,1) \subset \mathfrak{a}
$$

can be given as $\left\langle\Xi, \sqrt{-1} W_{I}\right\rangle$. The weight decomposition on $H^{\bullet}(M)$ associated with this Cartan algebra action coincides with the Hodge decomposition.

\section{A.3 Lie algebra generated by all $\mathfrak{s o}(4,1)$.}

The following was observed in [V1] (see also [LL]).

Proposition A.9: Let $\mathrm{L}_{\omega}, \Xi, \Lambda_{\omega}$ and $\mathrm{L}_{\omega^{\prime}}, \Xi, \Lambda_{\omega^{\prime}}$ be two $\mathfrak{s l}(2)$-triples on a hyperkähler manifolds. Then $\left[\Lambda_{\omega^{\prime}}, \Lambda_{\omega}\right]=0$.

Proof: From the Torelli theorem for hyperkähler manifolds (see e. g. $[\mathrm{AV}])$ it follows that the set of pairs $\omega_{I}, \omega_{J}$ associated with the hyperkähler structures is Zariski dense in the space of all pairs $\omega_{1}, \omega_{2} \in H^{2}(M, \mathbb{R})$ such that $q\left(\omega_{1}\right)=q\left(\omega_{2}\right)>0$ and $q\left(\omega_{1}, \omega_{2}\right)=0$. Therefore it suffices to prove 
N. Kurnosov, A. Soldatenkov, M. Verbitsky Kuga-Satake construction and cohomology of HK manifolds

this relation for $\omega, \omega^{\prime} \in\left\langle\omega_{I}, \omega_{J}, \omega_{K}\right\rangle$. In this case, the relation $\left[\Lambda_{\omega^{\prime}}, \Lambda_{\omega}\right]=0$ follows from commutation relations in $\mathfrak{s o}(4,1)$.

From this result, the following structure theorem can be deduced ([V2], $[\mathrm{LL}])$.

Theorem A.10: The algebra $\mathfrak{g}$ generated by all $\mathfrak{s o}(4,1)$ for all hyperkähler triples on a given hyperkähler manifold $M$ of maximal holonomy is isomorphic to $\mathfrak{s o}\left(4, b_{2}(M)-2\right)$.

Proof. Step 1: Consider the action of $\mathfrak{g}$ on the Mukai extension of $H^{2}(M, \mathbb{R})$ (see Section 3.4):

$$
\tilde{H}:=\mathbb{R} \cdot x \oplus H^{2}(M, \mathbb{R}) \oplus \mathbb{R} \cdot y,
$$

where $x$ has degree $0, y$ has degree $4, H^{2}(M, \mathbb{R})$ is in degree 2 . On $\tilde{H}$ we have the extended quadratic form $\tilde{q}$. The action of $\mathfrak{g}$ is determined by the following properties:

1. It is compatible with the grading;

2. For all $\alpha, \beta \in H^{2}(M, \mathbb{R})$, one has $\mathrm{L}_{\alpha} x=\alpha, \mathrm{L}_{\alpha} \beta=q(\alpha, \beta) y$, where $q$ is the $\mathrm{BBF}$ form.

3. $\Lambda_{\alpha} y=\alpha, \Lambda_{\alpha} \beta=q(\alpha, \beta) x$.

To see that this action is well-defined, we need to check that commutator relations hold. This follows from commutator relations in $\mathfrak{s o}(4,1)$ and Zariski density of pairs $\alpha, \beta \in\left\langle\omega_{I}, \omega_{J}, \omega_{K}\right\rangle$ in the set of all pairs $\alpha, \beta \in H^{2}(M, \mathbb{R})$. To obtain that the set of such pairs is Zariski dense, we use Torelli theorem (see e. g. $[\mathrm{AV}])$.

Step 2: We have constructed a homomorphism $\mathfrak{g} \longrightarrow \operatorname{End}(\tilde{H})$. By construction, it preserves the Mukai pairing $\tilde{q} \in S^{2}\left(\tilde{H}^{*}\right)$. This defines a homomorphism $\Psi: \mathfrak{g} \longrightarrow \mathfrak{s o}(\tilde{H}, \tilde{q})=\mathfrak{s o}\left(4, b_{2}(M)-2\right)$.

Step 3: $\Psi$ is surjective, because it is surjective on generators, and injective, because the relations in $\mathfrak{s o}\left(4, b_{2}-2\right)$ can be obtained from relations in $\mathfrak{s o}(4,1)$. 
N. Kurnosov, A. Soldatenkov, M. Verbitsky Kuga-Satake construction and cohomology of HK manifolds

Remark A.11: It is easy to see that the center of the corresponding Lie group $\operatorname{Spin}\left(4, b_{2}-2\right)$ acts as -1 on odd-dimensional cohomology and trivially on the even-dimensional cohomology (see [V3]).

Acknowledgement: Part of this work was done by A.S. and M.V. during the SCGP workshop on hyperkähler manifolds in September 2016. We are grateful to the organizers for the invitation and to the SCGP for perfect working conditions.

\section{References}

[AV] Amerik, E., Verbitsky, M., Teichmuller space for hyperkahler and symplectic structures, preprint, arXiv:1503.01201, 2015.

[Bes] Besse, A., Einstein Manifolds, Springer-Verlag, New York (1987)

[Bo1] Bogomolov, F., On the decomposition of Kähler manifolds with trivial canonical class, Math. USSR-Sb. 22 (1974) 580 - 583.

[Bo2] Bogomolov, F., On the cohomology ring of a simple hyper-Kähler manifold (on the results of Verbitsky) Geom. Funct. Anal., Vol. 6, No. 4, 1996, pp. 612-618.

[C] Calabi, E., Metriques kähleriennes et fibrès holomorphes, Ann. Ecol. Norm. Sup. 12 (1979), 269-294.

[DMOS] Deligne, P., Milne, J.S., Ogus, A., Shih, K., Hodge cycles, motives, and Shimura varieties, Lecture Notes in Mathematics, Vol. 900, SpringerVerlag, 1982.

[CP] de Concini, C., Procesi, C., A Characteristic Free Approach to Invariant Theory, Adv. in Math., 21, 1976, pp. 330-354.

[DS] Dancer, A., Swann, A., Hypersymplectic manifolds. In: Recent developments in pseudo-Riemannian geometry, 97-111, ESI Lect. Math. Phys., Eur. Math. Soc., Zürich, 2008.

[FKS] Figueroa-O'Farrill, J. M., Koehl, C.. Spence, B., Supersymmetry and the cohomology of (hyper)Kähler manifolds, Nuclear Phys. B 503 (1997), no. $3,614-626$.

[F] Fujiki, A. On the de Rham Cohomology Group of a Compact Kähler Symplectic Manifold, Adv. Stud. Pure Math. 10 (1987) 105-165. 
N. Kurnosov, A. Soldatenkov, M. Verbitsky Kuga-Satake construction and cohomology of HK manifolds

[GHJ] Gross, M., Huybrechts, D., Joyce, D., Calabi-Yau manifolds and related geometries, Universitext, Lectures from the Summer School held in Nordfjordeid, June 2001, Springer-Verlag, Berlin, 2003, viii+239 pp.

[Hi] Hitchin, N. J., Hypersymplectic quotients, Acta Acad. Sci. Tauriensis, supplemento al numero 124 (1990), 169-180.

[How] Howe, R., Remarks on classical invariant theory, Trans. Amer. Math. Soc., Vol. 313, No. 2, 1989, pp. 539-570.

[JV1] Jardim, M., Verbitsky, M., Moduli spaces of framed instanton bundles on $\mathbb{C} P^{3}$ and twistor sections of moduli spaces of instantons on $\mathbb{R}^{4}$. Adv. Math. 227 (2011),1526-1538.

[JV2] Jardim, M., Verbitsky, M., Trihyperkähler reduction and instanton bundles on $\mathbb{C} P^{3}$, arXiv:1103.4431 (to appear in Compositio Math.)

[Ka] Kamada, H., Neutral hyper-Kähler structures on primary Kodaira surfaces, Tsukuba J. Math. 23 (2) (1999), 321-332. 100

[KS] Kuga, M., Satake, I., Abelian varieties attached to polarized K3-surfaces, Math. Ann., 169 (1967), pp. 239-242.

[LL] Looijenga, E., Lunts, V., A Lie algebra attached to a projective variety, Invent. Math. 129 (1997), no. 2, 361-412.

[LM] Lawson, H.B., Michelsohn, M.-L., Spin geometry, Princeton University Press, 1989.

[SV2] Soldatenkov, A., Verbitsky, M., k-symplectic structures and absolutely trianalytic subvarieties in hyperkähler manifolds, J. Geom. Phys. 92 (2015), $147-156$.

[V0] Verbitsky, M., On the action of the Lie algebra $\mathfrak{s o ( 5 )}$ on the cohomology of a hyperkähler manifold, Func. Anal. and Appl. 24 (1990), 70-71.

[V1] Verbitsky, M., Cohomology of compact hyperkähler manifolds. alg-geom electronic preprint 9501001, 89 pages, LaTeX.

[V2] Verbitsky, M., Cohomology of compact hyperkähler manifolds and its applications, GAFA vol. 6 (4) pp. 601-612 (1996).

[V3] Verbitsky, M., Mirror Symmetry for hyperkähler manifolds, alggeom/9512195, Mirror symmetry, III (Montreal, PQ, 1995), 115-156, AMS/IP Stud. Adv. Math., 10, Amer. Math. Soc., Providence, RI, 1999.

[V4] Verbitsky, M., Hyperkähler manifolds with torsion, supersymmetry and Hodge theory, Asian J. of Math., Vol. 6 (4), pp. 679-712, December 2002. 
N. Kurnosov, A. Soldatenkov, M. Verbitsky Kuga-Satake construction and cohomology of HK manifolds

[Y] Yau, S. T., On the Ricci curvature of a compact Kähler manifold and the complex Monge-Ampère equation I. Comm. on Pure and Appl. Math. 31, 339-411 (1978).

NiKON KuRNOSOV

Laboratory of Algebraic Geometry,

National Research University HSE,

Department of Mathematics, 6 Usacheva Str. Moscow, Russia

ALSO:

DEPARTMENT OF MATHEMATICS,

UnIVERSITY OF GEORGIA,

1023 D. W. Brooks Drive, Athens, GA 30602, USA

Andrey Soldatenkov

Institut Für Mathematik, Humboldt-Universitätt ZU Berlin, UNTER DEN LiNDEN 6, 10099 BeRLin

soldatea@hu-berlin.de

Misha Verbitsky

Instituto Nacional de Matemática Pura e Aplicada

Estrada Dona Castorina, 110

JARDim BotÂNICO, CEP 22460-320

Rio de Janeiro, RJ - Brasil

ALSO:

Laboratory of Algebraic Geometry, National Research University HSE,

Department of Mathematics, 6 Usacheva Str. Moscow, Russia, verbit@impa.br, 